\title{
Analysis of Power Ramping Schemes for UTRA-FDD Random Access Channel
}

\author{
Yang Yang, Member, IEEE, and Tak-Shing Peter Yum, Senior Member, IEEE
}

\begin{abstract}
The random access channel (RACH) in a universal terrestrial radio access-frequency division duplex (UTRA-FDD) system is a contention-based channel mainly used to carry control information from mobile stations (MS) to base stations (BS). The transmission of a random access request contains two steps: preamble transmission and message transmission. In preamble transmission, the power ramping technique is used to favor the delayed preambles by stepping up the transmission power after each unsuccessful access. In doing so, the success of transmitting a long-delayed preamble is increased due to the power capture effect. This paper analyzes the blocking, throughput, and delay performance of preamble transmission under three power ramping schemes with fixed, linear, and geometric step sizes. The interference caused by different power ramping schemes is also compared.
\end{abstract}

Index Terms-Power ramping, random access channel (RACH), third-generation (3G) wireless communications, UTRA-FDD.

\section{INTRODUCTION}

$\mathbf{I}$ $\mathrm{N}$ UNIVERSAL terrestrial radio access-frequency division duplex (UTRA-FDD) of third-generation (3G) wireless communication systems, random access channel (RACH) is defined as a contention-based transport channel that is mainly used to carry control information, e.g., random access request, from mobile stations (MS) to base stations (BS) [1], [2]. The transmission of a random access request (packet) contains two steps.

1) Preamble Transmission: A preamble is of length 4096 chips and consists of 256 repetitions of an orthogonal spreading code of length 16 chips. To take advantage of the power capture effect, the power ramping technique, whereby the transmission power is increased by one step after every unsuccessful attempt, is used to increase the success probability of preamble retransmissions.

2) Message Transmission: A message is of length 10 or $20 \mathrm{~ms}$ and consists of two parts: a) the data part that carries the random access request or short data packet, and b) the control part that carries the pilot bits and rate information for the data part. These two parts are transmitted in parallel at power levels higher than that used in the last preamble transmission.

Manuscript received April 20, 2004; revised August 20, 2004; accepted November 21, 2004. The editor coordinating the review of this paper and approving it for publication is X. Shen. This work was supported in part by the Hong Kong Research Grants Council under Grant CUHK 4325/02E.

Y. Yang is with the Department of Electronic and Electrical Engineering, University College London (UCL), London WC1E 6BT, U.K. (e-mail: y.yang@ee.ucl.ac.uk).

T.-S. P. Yum is with the Department of Information Engineering, The Chinese University of Hong Kong, Shatin, New Territories, Hong Kong (e-mail: yum@ie.cuhk.edu.hk).

Digital Object Identifier 10.1109/TWC.2005.857993
The protocol used in RACH is slotted ALOHA with the length of an access slot equal to $4 / 3 \mathrm{~ms}$. Two frames (20 ms), or 15 access slots, constitute an access period. An access period is divided into two access slot sets (ASS): ASS-1 consists of the first eight access slots and ASS-2 consists of the remaining seven [2]. Before each access period, the BS broadcasts to all MSs the available access slots (maximum 15) in the next access period, the idle orthogonal codes (maximum 16) in each available slot, the transmission power range, the maximum number of retransmissions, and the power control parameters. An MS with a random access request to transmit selects at random an available access slot from the next ASS and a code from the available code set for the initial preamble transmission. ${ }^{1}$ The preamble transmission result is announced on the acquisition indicator channel (AICH). If the preamble is not correctly received by the $\mathrm{BS}$, the MS will retransmit another preamble using a new code at a higher power level after a random backoff delay. This access process continues until 1) a preamble retransmission is correctly received by the BS, or 1) the allowable number of retransmissions is reached. If case 1) occurs, the MS will transmit the message after three or four access slots. If it is successful, the random access procedure ends and the selected code is assigned to the MS as a dedicated channel (DCH). If the message transmission fails or case 2) occurs, the random access request is blocked and the MS may regenerate the request after some delay [3].

In this paper, we study three power ramping schemes (with fixed, linear, and geometric step sizes) and analyze their effects on the blocking, throughput, and delay performance in preamble transmission under a frequency-selective multipath Rayleigh slow-fading channel. The following section reviews the previous related work in literature. The system model, power capture model, and different power ramping schemes are presented in Section II. Based on these models and schemes, we derive in Section III success probability, throughput, average received power level, blocking probability, and access delay in preamble transmission. Different power ramping schemes are compared in Section IV.

\section{A. Related Work}

Under the nonblocking assumption (infinite buffer size) and additive white Gaussian noise (AWGN) channel model, the throughput and average delay performance of RACH was investigated analytically with and without power ramping in [4]. The delay capture effect on performance was also studied. Based on different physical channel models and parameters, simulation results on the performance of UTRA-FDD RACH

\footnotetext{
${ }^{1}$ In case there is no available slot in the next ASS, the preamble transmission will be delayed to the following ASS period.
} 
were reported in [5]-[7]. System performance can be further enhanced by the multi-threshold detection algorithm [8] and by the extra code selection before the following message transmission [9]. Different priority differentiation methods were proposed in [10]-[12]. Specifically, three access priority schemes, which can offer different delay performance for different traffic classes, were proposed in [10]. In [11], access slots and spreading codes are grouped for different traffic classes. As a result, the class with the largest group will have the highest success probability. In [12], different power-ramping step values are used for different traffic classes so that the class with a larger step size (high priority) will have higher throughput and shorter delay. All the previous work considered only constant power increment step size. This may incur many unnecessary retransmissions when the initial transmission power is low and the step size is small [13]. On the other hand, if a large step size is used, the average transmission power is higher. This causes a reduction of power capture probability and a higher interference level to the ongoing traffic.

\section{Problem Formulation}

The arrival of composite preamble transmissions (including initial transmissions and retransmissions) is modeled by a Poisson process with the rate $G$, which is also known as the offered traffic. Let $N$ be the number of MSs contending for the same access slot. The distribution of $N$ is therefore

$$
P\{N=n\}=\frac{G^{n} e^{-G}}{n !}, \quad n=0,1,2, \ldots
$$

For a given offered traffic $G$, let $S$ be the corresponding throughput of random access requests. Upon successfully receiving a random access request, i.e., both the preamble and the message transmissions are successful, the BS will assign the selected code as a DCH to the corresponding MS for its data packet transfer. Let $Q$ be the number of available codes in a typical access slot. The distribution of $Q$ can be derived as

$$
P\{Q=q\}=\frac{\pi_{0}}{1-\pi_{0}} \frac{16 !}{(16-q) ! S^{q}}, \quad 1 \leq q \leq 16
$$

where

$$
\pi_{0}=\left[\sum_{i=0}^{16} \frac{16 !}{(16-i) ! S^{i}}\right]^{-1} .
$$

Let $K$ be the number of MSs selecting the same code for accessing this slot. Given $N=n$ and $Q=q$, the probability that a tagged MS, say MS- $A$, is code collided with $(k-1)$ other MSs in a typical access slot is given by

$$
\begin{aligned}
P\{K & =k \mid N=n, Q=q\} \\
& =\left(\begin{array}{l}
n-1 \\
k-1
\end{array}\right)\left(\frac{1}{q}\right)^{k-1}\left(\frac{q-1}{q}\right)^{n-k}, \quad 1 \leq k \leq n .
\end{aligned}
$$

The physical channel under consideration is a frequencyselective multipath Rayleigh slow-fading channel. Assuming the shadowing and attenuation effects can be compensated by the open-loop power control used in RACH [14], the envelope of the received signal in one path is therefore a Rayleigh random variable. If a perfect RAKE receiver with $L$ fingers is used at the BS, the signal powers distributed in $L$ independent paths can be aggregated together so that the total received power $P$ has a gamma distribution, i.e.,

$$
f_{P}(x)=\frac{x^{L-1} \exp \left(-\frac{x}{\mu}\right)}{(L-1) ! \mu^{L}}, \quad x>0
$$

where $\mu$ is the average received power from each path.

\section{A. Power Capture Model}

Consider a typical access slot where there are $n$ simultaneous MSs, $q$ available codes, and the tagged MS, say MS- $A$, is code collided with $(k-1)$ MSs. Assume that the closed-loop power control used in DCH is accurate and the received powers from different DCHs are of the same value, say $\eta$. The total interference caused by $\mathrm{DCH}$ is therefore equal to $(16-q) \eta$. Comparing to this interference and the multiple access interference (MAI), the additive channel noise and self-interference are negligible [14].

A preamble contains only the repetitions of a selected code. By using a perfect RAKE receiver, the BS can aggregate the preamble transmissions from those $k$ code-collided MSs (including MS- $A$ ) [9], [15]. Therefore, the condition for correct reception of the preamble sent by MS- $A$ is

$$
\frac{P_{A}+\sum_{i=2}^{k} P_{i}}{(16-q) \eta+\sum_{i=k+1}^{n} P_{i}} \geq \beta_{1}
$$

where $P_{i}$ is the total received power from MS- $i$ and $\beta_{1}$ is the minimum signal-to-interference ratio (SIR) required for successfully decoding a preamble.

\section{B. Power Ramping Schemes}

By using open-loop power control, an MS can adjust its transmission power based on the received signal strength from the BS. The aim is to make the received signal at the BS exceed the preestimated, or target, power level. For the initial preamble transmission, we assume that all MSs have the same target power level $\mu \mathrm{L}$. If the initial preamble transmission fails, a higher target power level, decided by a particular power ramping scheme, will be used for retransmission. As in [16], we use $\mu \mathrm{L}$ as the power increment unit. Let $\left(m_{r} \mu \mathrm{L}\right)$ be the target power level in the $r$ th preamble retransmission. We study the following three power ramping schemes:

- fixed ramping: the power increment is fixed at one unit each time, i.e., $m_{r}=r+1$;

- linear ramping: the power increment is in steps of $1,2,3$, $4, \ldots$, units each time, i.e., $m_{r}=\left(r^{2}+r+2\right) / 2$;

- geometric ramping: the power increment is in steps of 1 , $2,4,8, \ldots$, units each time, i.e., $m_{r}=2^{r}$. 




Fig. 1. Flow diagram of random access request.

\section{Performance AnAlysis}

\section{A. Throughput}

Given that $N=n, Q=q$, and $K=k$, the conditional success probability of the $r$ th preamble retransmission, denoted by $u(r \mid n, q, k)$, can be derived according to the criterion (6) for correct reception of a preamble, i.e.,

$$
\begin{aligned}
& u(r \mid n, q, k) \\
& =\left\{\begin{array}{cl}
\frac{\exp \left(-\frac{\beta_{1} c}{\mu}\right)}{\left(1+\beta_{1}\right)^{b}(b-1) !} \sum_{i=0}^{a-1} \sum_{j=0}^{i} \frac{(b+j-1) !}{j !(i-j) !} & 1 \leq k \leq n-1 \\
\times \frac{\beta_{1}^{i}}{\left(1+\beta_{1}\right)^{j}}\left(\frac{c}{\mu}\right)^{i-j}, & k=n \\
\exp \left(-\frac{\beta_{1} c}{\mu}\right) \sum_{i=0}^{a-1} \frac{1}{i !}\left(\frac{\beta_{1} c}{\mu}\right)^{i}, & k=n
\end{array}\right.
\end{aligned}
$$

where the three interim variables $a, b$, and $c$ are defined as

$$
\left\{\begin{array}{l}
a=\left\lfloor(k-1) \bar{m} L+m_{r} L\right\rfloor \\
b=\lfloor(n-k) \bar{m} L\rfloor \\
c=(16-q) \eta .
\end{array}\right.
$$

In (8), $\bar{m}$ is the average target power level in the unit of $\mu \mathrm{L}$ and $\lfloor x\rfloor$ denotes the floor function. Removing the conditioning on $K, Q$, and $N$, we obtain

$$
\begin{array}{r}
u(r)=\sum_{n=1}^{\infty} \sum_{q=1}^{16} \sum_{k=1}^{n} u(r \mid n, q, k) P\{K=k \mid N=n, Q=q\} \\
\times P\{Q=q\} P\{N=n-1\} .
\end{array}
$$

Note that the success probability $u(r)$ of the $r$ th preamble retransmission is a function of $\bar{m}$. But $\bar{m}$ as expressed in (12) is a function of $u(r)$. Therefore, (9) and (12) are to be solved recursively.

The flow diagram of random access requests in $\mathrm{RACH}$ of UTRA-FDD is shown in Fig. 1. Let $G_{0}$ and $G_{r}$ denote, respectively, the arrival rates of the initial preamble transmissions and the $r$ th preamble retransmissions. Let $S_{P}$ be the throughput of preamble transmission and let $r_{\max }$ be the maximum number of retransmissions allowed. When RACH is in steady state, the following equations are satisfied:

$$
\left\{\begin{array}{l}
G_{0}=S_{P}+G_{r_{\max }+1} \\
G_{1}=G_{0}[1-u(0)] \\
G_{2}=G_{1}[1-u(1)] \\
\quad \vdots \\
G_{r_{\max }+1}=G_{r_{\max }}\left[1-u\left(r_{\max }\right)\right] .
\end{array}\right.
$$

The composite offered traffic is therefore $G=\sum_{r=0}^{r_{\max }} G_{r}$. The throughput of preamble transmission can be derived as

$$
S_{P}=G \frac{1-\prod_{i=0}^{r_{\max }}[1-u(i)]}{1+\sum_{r=1}^{r_{\max }} \prod_{i=0}^{r-1}[1-u(i)]} .
$$

The power ramping technique used in preamble transmission will generate much interference to the existing traffic. This effect can be measured by the average received (target) power level $\bar{m}$ at the BS. In the unit of $\mu \mathrm{L}, \bar{m}$ is given by

$$
\begin{aligned}
\bar{m} & =\sum_{r=0}^{r_{\max }} m_{r} \frac{G_{r}}{G} \\
& =\frac{1+\sum_{r=1}^{r_{\max }}\left\{m_{r} \prod_{i=0}^{r-1}[1-u(i)]\right\}}{1+\sum_{r=1}^{r_{\max }} \prod_{i=0}^{r-1}[1-u(i)]} .
\end{aligned}
$$

\section{B. Access Delay}

Let $R$ be the number of retransmissions needed before the preamble is successfully received by the BS. Under the assumption that all transmission attempts are independent, the distribution of $R$ is given by

$$
P\{R=r\}=u(r) \prod_{i=0}^{r-1}[1-u(i)], \quad r=0,1,2, \ldots
$$

As shown in Fig. 1, a preamble is blocked after $r_{\max }$ unsuccessful retransmissions. The blocking probability $P_{B}$ of preamble transmission is therefore

$$
P_{B}=P\left\{R>r_{\max }\right\}=\prod_{r=0}^{r_{\max }}[1-u(r)] .
$$

For successfully transmitted preambles, the distribution of their retransmissions $R^{\prime}$ is the distribution of $R$ conditioned on $R \leq r_{\max }$. In other words

$$
\begin{aligned}
P\left\{R^{\prime}=r\right\}= & P\left\{R=r \mid R \leq r_{\max }\right\} \\
= & \frac{u(r) \prod_{i=0}^{r-1}[1-u(i)]}{u(0)+\sum_{r=1}^{r_{\max }}\left\{u(r) \prod_{i=0}^{r-1}[1-u(i)]\right\}}, \\
& \quad r=0,1, \ldots, r_{\max } .
\end{aligned}
$$

As specified in [2], the random access slots are divided into $12 \mathrm{RACH}$ subchannels and these subchannels are further grouped into sets of different sizes. Each set of subchannels 


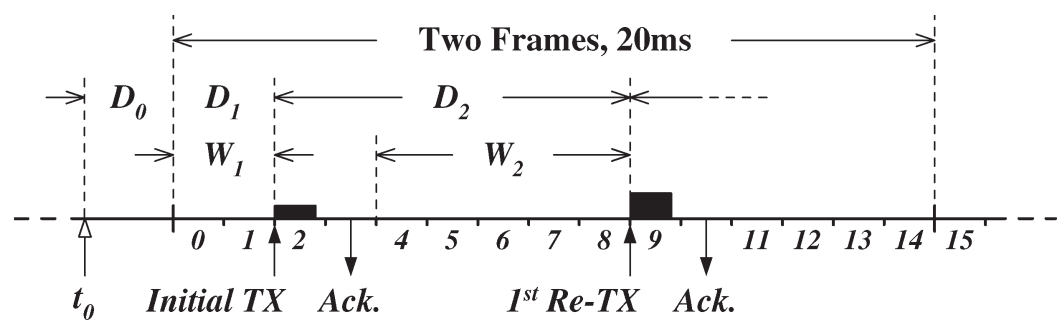

Fig. 2. Access mechanism of preamble transmission.

is associated with one access service class (ASC) and at most eight ASCs can be supported in RACH [3]. In this paper, we assume all random access requests are of the same ASC and all access slots are available for selection. ${ }^{2}$

Referring to Fig. 2, let $t_{0}$ be the time when a random access request is generated. After an average of 0.5 frame waiting time $D_{0}$, the random access request randomly selects an available slot from the next ASS for its initial preamble transmission. The skipped slots (in the same ASS) before that initial transmission constitute the first backoff delay $D_{1}$ (or $W_{1}$ ). The error-free positive acknowledgment can be received in the next slot from AICH. As the retransmission procedure specified in the UTRAFDD standard is complicated and analytically intractable [2], we choose the simple uniform backoff (UB) policy [17] with range $[0, \omega]$ for the study of delay performance under different power ramping schemes. When the initial preamble transmission is unsuccessful, the second random backoff delay $W_{2}$ is also drawn from $[0, \omega]$ and the first retransmission is arranged at the $\left(W_{2}+1\right)$ th access slot after receiving the first acknowledgment. Let $D_{i}=W_{i}+2\left(2 \leq i \leq r_{\max }+1\right)$ be the delay interval between the $(i-1)$ th and the $i$ th transmission attempts. Adding the one-slot transmission time, the total access delay $D_{P}$ (in unit of slots) for preamble transmission is given by

$$
\begin{aligned}
D_{P} & =1+D_{0}^{\prime}+\sum_{i=1}^{R^{\prime}+1} D_{i} \\
& =1+D_{0}^{\prime}+\sum_{i=1}^{R^{\prime}+1} W_{i}+2 R^{\prime}
\end{aligned}
$$

where $D_{0}^{\prime}$ is the normalized initial waiting time and is uniformly distributed in $(0,7.5$ ] (one frame is equal to 7.5 access slots in length).

The average access delay $E\left[D_{P}\right]$ and delay variance $\sigma_{D_{P}}^{2}$ for preamble transmission can then be derived as [17]

$$
\begin{aligned}
E\left[D_{P}\right] & =1+E\left[D_{0}^{\prime}\right]+\left(E\left[R^{\prime}\right]+1\right) E\left[W_{i}\right]+2 E\left[R^{\prime}\right] \\
& =4.75+\frac{\omega}{2}+\frac{\omega+4}{2} E\left[R^{\prime}\right]
\end{aligned}
$$

TABLE I

BASIC PARAMETER IN NUMERICAL EVAlUation

\begin{tabular}{|c|c|}
\hline \hline Parameter & Value \\
\hline \hline Number of Resolvable Paths & $L=2$ \\
\hline Average Received Power from One Path & $\mu=1$ \\
\hline Average Received Power from One DCH & $\eta=2$ \\
\hline Minimum SIR for Correct Preamble Reception & $\beta_{1}=-5 \mathrm{~dB}$ \\
\hline Maximum Allowed Retransmission Attempts & $r_{\max }=5$ \\
\hline Backoff Delay Range & $\omega=7$ \\
\hline
\end{tabular}

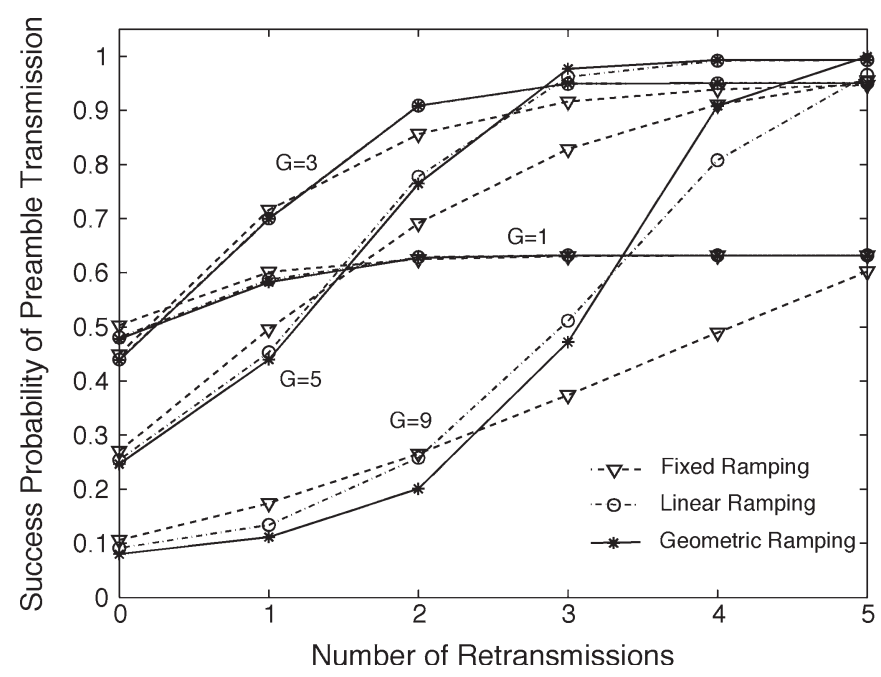

Fig. 3. Success probability of preamble transmission, $G=1,3,5$, and 9 .

and

$$
\begin{aligned}
\sigma_{D_{P}}^{2} & =\operatorname{Var}\left(D_{0}^{\prime}\right)+\operatorname{Var}\left(\sum_{i=1}^{R^{\prime}+1}\left(W_{i}+2\right)\right) \\
& =4.6875+\frac{\omega^{2}+2 \omega}{12}\left(E\left[R^{\prime}\right]+1\right)+\frac{(\omega+4)^{2}}{4} \operatorname{Var}\left(R^{\prime}\right)
\end{aligned}
$$

where the distribution of $R^{\prime}$ is given in (15).

\section{NUMERICAL RESUltS}

The parameters for numerical evaluation are listed in Table I.

${ }^{2}$ The probability that a slot is not available (i.e., it contains no idle spreading code) is equal to $\pi_{0}$. As implied by (3), $\pi_{0}$ is very small even when the system throughput is maximum.
Fig. 3 compares the success probability $u(r)$ of preamble transmission under different power ramping schemes and for 


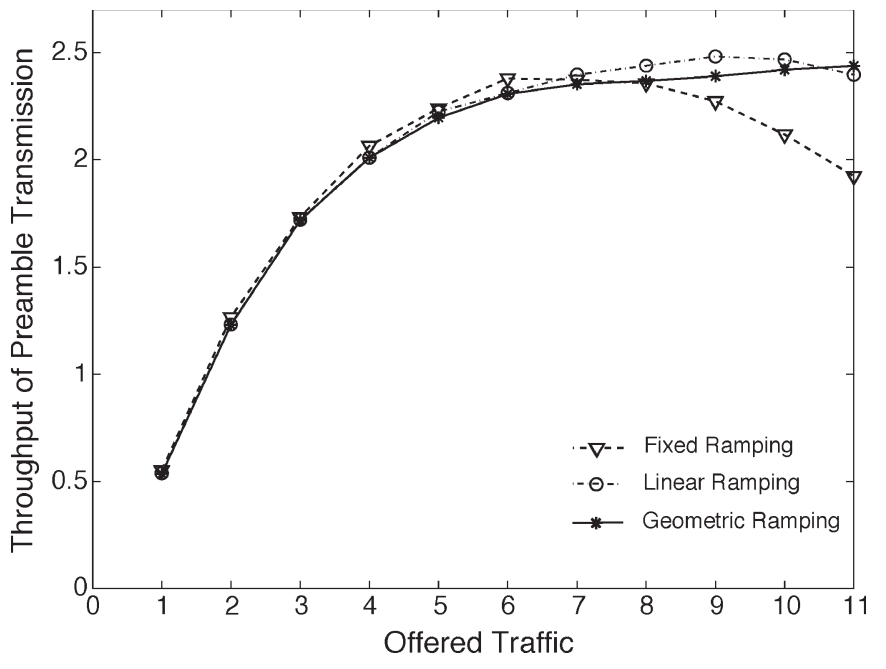

Fig. 4. Throughput of preamble transmission.

different offered traffic values. When the offered traffic is small, say $G=1$, power ramping can only improve the success probability slightly. Referring to (1) and (4), for small $G$ values, few preamble transmissions take place in the same slot and the chance for code collision is even less as most codes are available for selection. In this case, the number of simultaneous MS $N$ and the number of code-collided MS $K$ are mostly equal to one (collision free), and the number of available codes $Q$ is always close to the maximum value of 16 . Therefore, the probability $u(r \mid N=1, Q=16, K=1)$ is the dominating item in the calculation of $u(r)$. As implied by (7) and (8), $u(r \mid N=1, Q=16, K=1)$ is insensitive to $r$ (the number of retransmissions) and $m_{r}$ (the corresponding target power level). So, $u(r)$ is flat for $G=1$.

When the offered traffic becomes larger, the success probability $u(0)$ of the initial transmission decreases so that more MSs need to retransmit their preambles at higher power levels. As shown in the figure, all three power ramping schemes can effectively increase the success probability. Between them, geometric ramping and linear ramping have similar performance while fixed ramping is inferior.

The throughput of preamble transmission $S_{P}$ is shown in Fig. 4. The throughput curves are virtually the same for the three schemes except at very high $G$ values. The maximum throughput is about 2.5. This figure shows that linear and geometric ramping schemes are more reliable than the fixed ramping scheme when $G$ is large.

The average target power level $\bar{m}$ of preambles is a measurement of the interference caused by power ramping. Fig. 5 shows $\bar{m}$ as a function of $G$ for different schemes. As expected, fixed ramping has the lowest power level. Geometric ramping causes a very high interference to ongoing traffic when the offered traffic is large.

The blocking probability $P_{B}$ of preamble transmission is shown in Fig. 6. Referring to (14), $P_{B}$ is the product of $\left(r_{\max }+1\right)$ complementary success probabilities. When the offered traffic is small, say $G \leq 2$, the success probability $u(r)$ is insensitive to the target power level $m_{r}$ (see Fig. 3) so that the blocking probabilities are indistinguishable for the

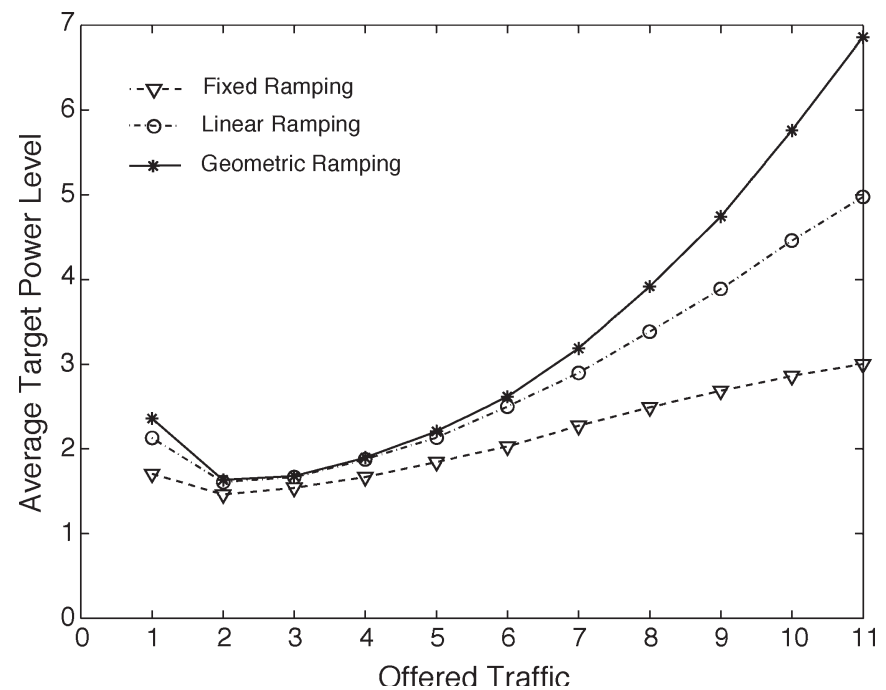

Fig. 5. Average target power level of preamble transmission.



Fig. 6. Blocking probability of preamble transmission.

three schemes. When the offered traffic becomes larger, say $G=3$, the success probability curves are greatly improved by different power ramping schemes. Therefore, the resulting blocking probabilities become smaller, which indicates that most preambles can be successfully transmitted by less than $\left(r_{\max }+1\right)$ attempts. When the offered traffic becomes much larger, say $G=9$, the success probabilities of the initial transmission and the first couple of retransmissions are quite small. As a result, more retransmissions at higher power levels are now needed for successfully transmitting a preamble. The blocking probabilities for different schemes are therefore increasing with respect to the offered traffic. Specifically, fixed ramping, linear ramping, and geometric ramping offer their minimum blocking probabilities when the offered traffic values are around 3,5, and 6 , respectively. Among the three schemes, geometric ramping has fast-increasing power increment steps so that it is the most effective scheme in enhancing success probability and offers the lowest blocking probability curve. 


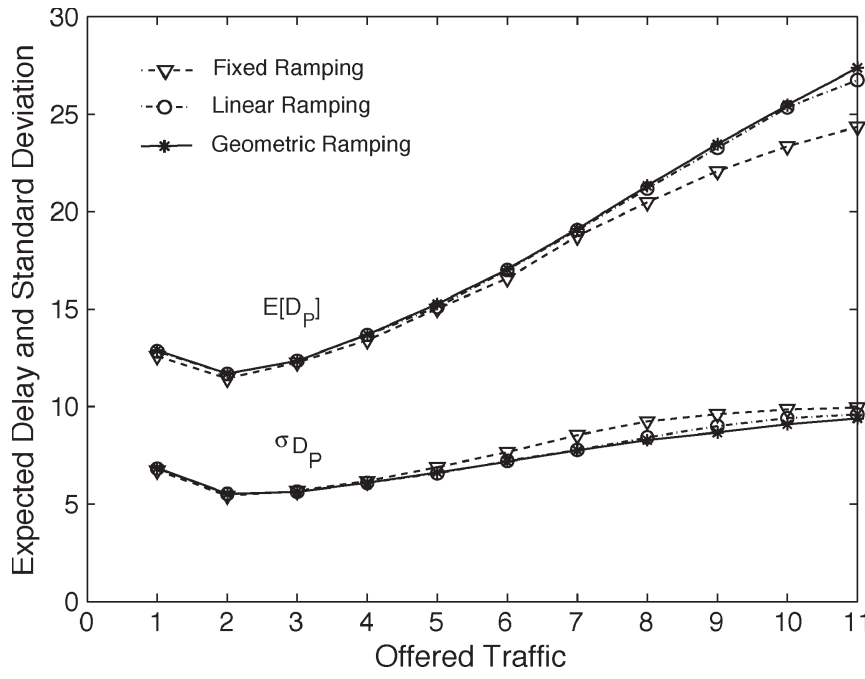

Fig. 7. Expected delay and delay standard deviation of preamble transmission.

Fig. 7 shows that the delay statistics of the three power ramping schemes are very close to each other. This is because all successful random access requests are transmitted within a small number of retransmissions $\left(r \leq r_{\max }=5\right)$. Therefore, the differences in $E\left[R^{\prime}\right]$ and $\operatorname{Var}\left(R^{\prime}\right)$, which are key parameters in the calculation of $E\left[D_{P}\right]$ and $\sigma_{D_{P}}$, given by the three power ramping schemes are indistinguishable for small $G$ values. It is seen the use of UB policy here is very "conservative" in spreading out the backlogged traffic in time and accounts for a fairly small delay standard deviation as shown.

\section{CONCLUSION}

Power ramping is used in the random access channel (RACH) of universal terrestrial radio access-frequency division duplex (UTRA-FDD) systems. We have derived in this paper, under three different power ramping schemes, success probability, throughput, average received power level, blocking probability, and access delay in preamble transmission. Our analysis considered a frequency-selective multipath Rayleigh slowfading channel, an ideal RAKE receiver, and the signal-tointerference ratio (SIR)-based power capture model. Numerical results showed that the linear and geometric ramping schemes are more effective than the fixed ramping scheme in increasing the success probability at the expense of high interference to existing traffic.

\section{REFERENCES}

[1] 3GPP TS 25.211 (V6.0.0), "Physical channels and mapping of transport channels onto physical channels (FDD)," Technical Specification (Release 6), Technical Specification Group Radio Access Network, Dec. 2003, 3GPP.

[2] 3GPP TS 25.214 (V6.0.0), "Physical layer procedures (FDD)," Technical Specification (Release 6), Technical Specification Group Radio Access Network, Dec. 2003, 3GPP.

[3] 3GPP TS 25.321 (V6.0.0), "Medium access control (MAC) protocol specification," Technical Specification (Release 6), Technical Specification Group Radio Access Network, Dec. 2003, 3GPP.

[4] S. S. Lim, Q. Cao, C. Demetrescu, D. J. Reader, and J. Lin, "3rd generation RACH transmission-A candidate," in Proc. IEEE Vehicular Technology Conf. (VTC), Amsterdam, The Netherlands, May 1999, vol. 1, pp. $140-144$.

[5] F. Khan, C. Roobol, and J. Larsson, "Performance of a common channel packet access in WCDMA," in Proc. IEEE Personal Indoor and Mobile Radio Communications (PIMRC), Boston, MA, Sep. 1998, vol. 1, pp. 198-202.

[6] M. Schulist and G. Frank, "Link level performance results for a WCDMA random access scheme with preamble power ramping and fast acquisition indication," in Proc. IEEE Vehicular Technology Conf. (VTC), Amsterdam, The Netherlands, Sep. 1999, vol. 5, pp. 2581-2585.

[7] I. N. Vukovic and T. Brown, "Performance analysis of the random access channel (RACH) in WCDMA," in Proc. IEEE Vehicular Technology Conf. (VTC), Rhodes, Greece, May 2001, vol. 1, pp. 532-536.

[8] Q. Zhang, M.-C. Chuah, and O.-C. Yue, "Enhanced power ramping scheme for UMTS random access channel," in Proc. IEEE Vehicular Technology Conf. (VTC), Amsterdam, The Netherlands, Sep. 1999, vol. 5, pp. 2631-2635.

[9] J. Moberg, M. Löfgren, and R. S. Karlsson, "Throughput of the WCDMA random access channel," in Proc. IST Mobile Communication Summit, Galway, Ireland, Oct. 2000. [Online]. Available: http://www. s3.kth.se/php/index.php?action $=$ publications \&year $=2000 \&$ author $=\mathrm{L} \%$ F6fgren $\&$ title $=\& \operatorname{div}=$ Radio \& type $=\&$ search $=$ yes

[10] M. C. Chuah, Q. Q. Zhang, and O. C. Yue, "Access priority schemes in UMTS MAC," in Proc. IEEE Wireless Communications and Networking Conf. (WCNC), New Orleans, LA, Sep. 1999, vol. 2, pp. 781-786.

[11] C.-L. Lin, "Investigation of 3rd generation mobile communication RACH transmission," in Proc. IEEE Vehicular Technology Conf. (VTC), Boston, MA, Sep. 2000, vol. 2, pp. 662-667.

[12] H.-D. Kim, S.-H. Wie, and D.-H. Cho, "A prioritized random access with discriminative power ramping step size," in Proc. IEEE Vehicular Technology Conf. (VTC), Boston, MA, Sep. 2000, vol. 4, pp. 1751-1757.

[13] R. Esmailzadeh and M. Gustafsson, "A new slotted ALOHA based random access method for CDMA systems," in Proc. IEEE 6th Int. Conf. Universal Personal Communications, San Diego, CA, Oct. 1997, vol. 1, pp. 43-47.

[14] N. Kong and L. B. Milstein, "Error probability of multicell CDMA over frequency selective fading channels with power control error," IEEE Trans. Commun., vol. 47, no. 4, pp. 608-617, Apr. 1999.

[15] D. H. Davis and S. A. Gronemeyer, "Performance of slotted ALOHA random access with delay capture and randomized time of arrival," IEEE Trans. Commun., vol. COMM-28, no. 5, pp. 703-710, May 1980.

[16] C. C. Lee, "Random signal levels for channel access in packet broadcast networks," IEEE J. Sel. Areas Commun., vol. SAC-5, no. 6, pp. 1026-1034, Jul. 1987.

[17] Y. Yang and T.-S. P. Yum, "Delay distributions of slotted ALOHA and CSMA," IEEE Trans. Commun., vol. 51, no. 11, pp. 1846-1857, Nov. 2003. 